\title{
Dysgranulopoiesis in patients with coronavirus disease 2019
}

\author{
Carolin Riemer ${ }^{1}$, Elisabeth Mack ${ }^{1}$, Andreas Burchert ${ }^{1}$, Margrit Gündisch ${ }^{2}$, Sarah Greib ${ }^{2}$, \\ Rojin Etati ${ }^{1}$, Thomas Tarawneh ${ }^{1}$, Ihab Karim ${ }^{1}$, Christian Keller ${ }^{3}$, Harald Renz ${ }^{2}$, \\ Chrysanthi Skevaki ${ }^{2}$, Andreas Neubauer ${ }^{1}$, Christian Michel ${ }^{1 *}$ \\ ${ }^{1}$ Department of Hematology, Oncology, Immunology, Philipps University Marburg, Germany \\ ${ }^{2}$ Institute of Laboratory Medicine, Universities of Giessen and Marburg Lung Center (UGMLC), Philipps University Marburg, \\ German Center for Lung Research (DZL), Marburg, Germany \\ ${ }^{3}$ Institute of Virology, Philipps University Marburg and University Hospital Giessen and Marburg, \\ Marburg, Germany
}

\section{Introduction}

The coronavirus disease 2019 (COVID-19) caused by severe acute respiratory syndrome coronavirus 2 (SARS-CoV-2) was first identified in a group of patients with acute pneumonia in Wuhan, the capital of Hubei Province in the People's Republic of China, in late December 2019 [1, 2]. While COVID-19 predominantly affects the lungs, it is not limited to respiratory manifestations, and has the potential to affect numerous organs including the hematopoietic system [3].

In addition to quantitative changes in the complete blood cell count, several publications report multi-lineage morphological abnormalities in peripheral blood cells during COVID-19 infection. A leucoerythroblastic reaction characterized by the appearance of myelocytes and nucleated red blood cells is frequently seen in COVID-19-patients. Erythrocytes show distinct aberrations of erythropoiesis including basophilic stippling and anisocytosis [4-6]. In addition, the absence of nuclear segmentation in neutrophils consistent with pseudo Pelger morphology has been reported in many cases. Further abnormalities regarding neutrophils involve ring-shaped nuclei, clumped chromatin, disintegrated apoptotic cell formations, cytoplasmic vacuoles, and dark toxic granulation $[5,6]$.

COVID-19 patients typically show a range of reactive lymphocytes including lymphoplasmacytoid cells, an increased proportion of large granular lymphocytes (LGL) as well as circulating plasma cells and mott cells [4-8]. Activated monocytes with aberrant nuclei, clumped chromatin and prominent vacuolization have been reported frequently $[4,5]$. The appearance of circulating megakaryocytes, and especially giant platelets with occasional focal attachment to the surface of leukocytes, represent additional COVID-19-associated findings in peripheral blood films and have been described in several publications and case reports $[4,5]$. In our study, we aimed to investigate specific morphological characteristics of neutrophil granulocytes in COVID-19 patients in direct comparison with control patients negative for SARS-CoV-2, but with clinical presentations strongly suggestive of COVID-19.

\section{Material and methods}

\section{Patients}

This study included 42 patients who were admitted to our hospital with respiratory symptoms during the first wave of COVID-19 between April and June 2020. Patients were eligible for the study if a diagnostic test to confirm, or conversely rule out, COVID-19 [SARS-CoV-2-polymerase chain reaction (PCR) or chest computed tomography scan] was performed at initial presentation.

\section{SARS-CoV-2 polymerase chain reaction}

Isolation and purification of viral RNA from a nasopharyngeal swab were performed via the EZ1 Virus Mini Kit v2.0

\footnotetext{
*Address for correspondence: Christian Michel, Department of Hematology, Oncology, Immunology, Philipps University Marburg, Baldingerstrasse, 35043 Marburg, Germany, e-mail: an.christian.michel@gmx.de
}

\section{PTHiT Copyright $\odot 2021$}

The Polish Society of Haematologists and Transfusiologists, Insitute of Haematology and Transfusion Medicine. All rights reserved. 
and EZ1 Advanced XL device (Quiagen, Hilden, Germany) according to the manufacturer's recommendations.

Amplification of SARS-CoV-2 specific spike gene (S gene) and beta-coronavirus specific envelope gene (E gene) were done via RealStar SARS-CoV-2 RT-PCR Kit 1.0 (Altona Diagnostics, Hamburg, Germany) according to the manufacturer's protocol.

\section{Blood smears and morphological examination}

Blood smears were automatically performed by the Sysmex SP-50 system (Sysmex, Norderstedt, Germany) and automatically stained with undiluted May Gruenwald solution for 72 seconds, followed by staining with 1:10 diluted May Gruenwald solution for 24 seconds, and staining with 1:25 diluted Giemsa solution for 192 seconds. Slides were analyzed by light microscopy using an OLYMPUS BX-53 microscope (Olympus, Tokyo, Japan), oil objective $\times 100$, equipped with OLYMPUS cellSens standard version 2.3 software for image acquisition.

\section{Statistical analyses}

For comparison of hypogranularity between COVID-19 patients and control patients, Fisher's exact test was used and performed by GraphPadPrism software, version 9.0.0 (GraphPad Software Inc, San Diego, CA, USA). Required sample size of COVID-19 patients for significant correlation of hypogranularity and death with power $=0.8$ was computed by $\mathrm{G} *$ Power 3.1.9.4 (Heinrich-Heine University Duesseldorf, Duesseldorf, Germany).

\section{Ethical approval}

This study was approved by the local ethics committee (no. 57/20). Informed consent was obtained from all patients.

\section{Results and discussion}

\section{Patients for assessment of peripheral blood smears}

From April until June 2020, 42 patients were included with COVID-19 characteristic symptoms, such as cough, shortness of breath, or fever. 20 of these patients had a positive result of SARS-CoV-2 PCR from a nasal swab and were consequently regarded as the SARS-CoV-2 positive group. The other 22 patients, who formed the control group, presented in the same time period as the suspected cases of COVID-19, but did not have an infection with SARS-CoV-2, thus showing a negative SARS-CoV-2 PCR and an alternative explanation of the symptoms during follow up. Blood smears were available from 19 patients in the SARS-CoV-2-positive group and from 20 patients in the control group. Age and comorbidities were distributed equally within both groups. Patients' characteristics are shown in Table I.
Hypogranularity and other dysplastic features of neutrophilic granulocytes in patients with COVID-19

Several dysplastic and reactive alterations of neutrophilic granulocytes could be frequently observed in SARS-CoV-2-positive patients (Figures 1A, B): 18 of 19 patients (94.7\%) displayed hyposegmented neutrophils. Ring-shaped nuclei were prominent in 10 of 19 patients (52.6\%) and bizarre nuclear formations could be observed in 14 of 19 patients (73.7\%). Neutrophils from 1 of 19 patients (5.3\%) showed toxic granulations; Döhle bodies could be detected in 2 of 10 patients (10.6\%), 10 of 19 patients (52.6\%) displayed cytoplasmic vacuoles in neutrophils. Hypogranularity of neutrophilic granulocytes was observable in 9 of 19 patients (47.4\%).

Hypogranularity was significantly more frequent in patients positive for SARS-CoV-2 at diagnosis than in symptomatic controls ( $47.4 \%$ vs. $15 \%, p=0.0407$; Figure $1 \mathrm{C}$ ). This hypogranularity was reversible in 55.6 per cent of the cases during the inpatient hospital stay (Figure 1D) resulting in normal or strong neutrophilic granulations.

Median time of granularity normalization was 36 days. Interestingly, 4 of 9 patients (44.4\%) with hypogranular neutrophils died, whereas in the group of patients with normal granularity only 1 of 10 patients (10\%) died. This effect was not statistically significant ( $p=0.1409)$. Computed sample size of COVID-19 patients required for a significant correlation of hypogranularity and death was $n=40$.

Increased concentrations of neutrophilic granulocytes have recently been described as a typical COVID-19 associated phenomenon [9-11]. Neutrophils are significantly involved in systemic hyperinflammation [12, 13]. Previous analyses showed morphological alterations and dysplastic features of neutrophilic granulocytes in COVID-19 patients $[5,6,8,14]$. In our study, we found that hypogranularity of neutrophilic granulocytes is significantly more frequent in COVID-19 patients than in control patients with comparable clinical features. Neutrophil degranulation in general is a feature of many inflammatory disorders [15].

Thus, in the context of COVID-19, hypogranularity is probably linked to systemic hyperinflammation and the attempt to eliminate the virus. Interestingly, the observed hypogranularity was reversible in $56 \%$ of our patients. This dynamic change has not previously been described. The findings of our study underscore a severe, but reversible, perturbation of granulopoiesis by SARS-CoV-2.

Although larger patient numbers and further analyses would be needed for confirmation, our results might indicate that granulocytic function in COVID-19 is affected more severely than in other reactive conditions. The presence of hypogranular neutrophils may be associated with dismal outcome, even though this effect was not statistically significant in our small patient cohort. Further studies will be needed to elucidate the involvement of granulopoiesis in the evolution of COVID-19. 
Table I. Patient characteristics

\begin{tabular}{|c|c|c|}
\hline Variable & CoVID-19 patients $(n=20)$ & Control patients $(n=22)$ \\
\hline Demographic characteristics & \multicolumn{2}{|c|}{ Median $(95 \% \mathrm{Cl})$} \\
\hline \multirow[t]{2}{*}{ Age (years) } & $66.5(62.0-75.0)$ & $70(58.0-80.0)$ \\
\hline & \multicolumn{2}{|c|}{ No. [\%] } \\
\hline Male & $16(80.0)$ & $12(54.5)$ \\
\hline Comorbidities & \multicolumn{2}{|c|}{ No. [\%] } \\
\hline Any comorbidity & $19(95.0)$ & $21(95.5)$ \\
\hline Former or current smoker & $2(10.0)$ & $8(36.4)$ \\
\hline Hypertension & $14(70.0)$ & $14(63.6)$ \\
\hline Obesity (BMI $\geq 30 \mathrm{~kg} / \mathrm{m}^{2}$ ) & $9(45.0)$ & $4(18.2)$ \\
\hline Overweight (BMI $\geq 25 \mathrm{~kg} / \mathrm{m}^{2},<30 \mathrm{~kg} / \mathrm{m}^{2}$ ) & $7(35.0)$ & $2(9.1)$ \\
\hline Diabetes & $7(35.0)$ & $2(9.1)$ \\
\hline Hyperlipoproteinemia & $3(15.0)$ & $2(9.1)$ \\
\hline Chronic heart disease & $8(40.0)$ & $14(63.6)$ \\
\hline Peripheral arterial disease & $0(0.0)$ & $2(9.1)$ \\
\hline Chronic obstructive pulmonary disease (COPD) & $0(0.0)$ & $4(18.2)$ \\
\hline Asthma & $1(5.0)$ & $0(0.0)$ \\
\hline Chronic renal disease & $3(15.0)$ & $7(31.8)$ \\
\hline Rheumatic diseases & $2(10.0)$ & $1(4.5)$ \\
\hline Neurological and neuromuscular diseases & $8(40.0)$ & $7(31.8)$ \\
\hline Malignant tumor & $5(25.0)$ & $6(27.3)$ \\
\hline Immunodeficiency & $4(20.0)$ & $3(13.6)$ \\
\hline Liver disease & $2(10.0)$ & $3(13.6)$ \\
\hline Thyroid disease & $2(10.0)$ & $4(18.2)$ \\
\hline Gastrointestinal disease & $8(40.0)$ & $6(27.3)$ \\
\hline Allergic disease & $3(15.0)$ & $1(4.5)$ \\
\hline Mental illness & $2(10.0)$ & $6(27.3)$ \\
\hline Addiction & $3(15.0)$ & $2(9.1)$ \\
\hline Current medication & \multicolumn{2}{|c|}{ No. [\%] } \\
\hline Antidiabetic drugs & $7(35.0)$ & $1(4.5)$ \\
\hline Angiotensin converting-enzyme inhibitors (ACE inhibitors) & $6(30.0)$ & $4(18.2)$ \\
\hline Beta-blockers & $7(35.0)$ & $11(50.0)$ \\
\hline Angiotensin receptor blockers (ARBs) & $2(10.0)$ & $3(13.6)$ \\
\hline Lipid-lowering drugs & $4(20.0)$ & $5(22.7)$ \\
\hline Platelet agglutination inhibitors & $5(25.0)$ & $7(31.8)$ \\
\hline Direct oral anticoagulants (DOACs) & $3(15.0)$ & $4(18.2)$ \\
\hline Phenprocoumon & $0(0.0)$ & $0(0.0)$ \\
\hline Calcium channel blockers (CCBs) & $4(20.0)$ & $3(13.6)$ \\
\hline Diuretics & $4(20.0)$ & $11(50.0)$ \\
\hline Systemic corticosteroids & $0(0.0)$ & $5(22.7)$ \\
\hline Anti-infective drugs (antibiotic, antiviral and antifungal medication) & $2(10.0)$ & $2(9.1)$ \\
\hline Analgesic & $3(15.0)$ & $8(36.4)$ \\
\hline Proton-pump inhibitors (PPIs) & $8(40.0)$ & $8(36.4)$ \\
\hline Immunosuppressive drugs & $2(10.0)$ & $3(13.6)$ \\
\hline
\end{tabular}


Table I (cont.). Patient characteristics

Inhaled corticosteroids

Inhaled adrenergic $\beta_{2}$ receptor agonists

Inhaled anticholinergics

Thyroid drugs

Anticonvulsants

Psychotropic drugs

Antineoplastic drugs

\section{Symptoms at admission}

Fever

Cough

Shortness of breath

Abdominal pain

Nausea and vomiting

Diarrhea

Headache

Fatigue

Myalgia or arthralgia

Tachycardia

Edema

Deterioration of general condition

Vital signs at admission

Heart rate [/min]

Systolic blood pressure [mm Hg]

Diastolic blood pressure [mm $\mathrm{Hg}]$

Respiratory rate [/min]

Temperature $\left[{ }^{\circ} \mathrm{C}\right.$ ]

Need for supplemental oxygen

\section{Chest auscultation at admission}

Any abnormal breath sounds

Crackling

Diminished breath sounds

No pathological findings

\section{CT findings}

Abnormalities on chest CT consistent with viral pneumonia

\section{Treatment}

Admission to intensive care unit

Oxygen therapy or non-invasive ventilation

Invasive ventilation

Extracorporeal membrane oxygenation (ECMO)

Prone positioning

Catecholamines

№. [\%]

$1(5.0)$

$1(4.5)$

$1(5.0)$

$4(18.2)$

$0(0.0)$

$3(13.6)$

$1(5.0)$

$0(0.0)$

$4(18.2)$

$4(18.2)$

$3(15.0)$

$5(22.7)$

1 (5.0)

2 (9.1)

No. [\%]

$9(45.0)$

8 (40.0)

$9(45.0)$

1 (5.0)

2 (10.0)

$4(20.0)$

$1(5.0)$

2 (10.0)

$2(10.0)$

$1(5.0)$

$0(0.0)$

14 (63.6)

10 (45.5)

14 (63.6)

2 (9.1)

3 (13.6)

$0(0.0)$

$1(4.5)$

3 (13.6)

1 (4.5)

$1(4.5)$

5 (22.7)

$5(25.0)$

4 (18.2)

Median (95\% Cl)

84.5 (76.0-100.0)

139.0 (127.0-165.0)

78.5 (62.0-85.0)

$20.0(16.0-28.0)$

37.1 (36.8-37.8)

No. [\%]

$6(30.0)$

$6(30.0)$

$0(0.0)$

5 (25.0)

20 (100.0)

12 (60.0)

$11(55.0)$

1 (5.0)

$10(50.0)$

$13(65.0)$

No. [\%]

No. [\%]

No. [\%]
$90.0(77.0-100.0)$ 140.0 (128.0-156.0)

76.0 (70.0-92.0)

$19.0(16.0-26.0)$

$37.15(36.7-37.8)$

$11(50.0)$

5 (22.7)

$5(22.7)$

10 (45.5)

$12(54.5)$

14 (63.6)

5 (22.7)

$0(0.0)$

$1(4.5)$

$5(22.7)$ 
Table I (cont.). Patient characteristics

\begin{tabular}{|c|c|c|}
\hline Variable & CovID-19 patients $(n=20)$ & Control patients $(n=22)$ \\
\hline & \multicolumn{2}{|c|}{ No. [\%] } \\
\hline Hemodialysis & 7 (35.0) & $2(9.1)$ \\
\hline Antibiotic treatment & $17(85.0)$ & $19(86.4)$ \\
\hline Antiviral treatment & $5(25.0)$ & $4(18.2)$ \\
\hline Antifungal treatment & $3(15.0)$ & $1(4.5)$ \\
\hline Treatment duration & \multicolumn{2}{|c|}{ Median $(95 \% \mathrm{Cl})$} \\
\hline Length of hospital stay (days) & $28.5(19.0-39.0)$ & $13.5(8.0-23.0)$ \\
\hline Duration of ventilation (days) & $27.0(18.0-41.0)$ & $4.0(2.0-9.0)$ \\
\hline Complications & \multicolumn{2}{|c|}{ No. [\%] } \\
\hline Respiratory complications & $17(85.0)$ & $17(77.3)$ \\
\hline Sepsis & $16(80.0)$ & $19(86.4)$ \\
\hline Organ dysfunction & $17(85.0)$ & $10(45.5)$ \\
\hline Electrolyte imbalance & $10(50.0)$ & $10(45.5)$ \\
\hline Clinical outcome & \multicolumn{2}{|c|}{ No. [\%] } \\
\hline Discharged & $16(80.0)$ & 21 (95.5) \\
\hline Died & $4(20.0)$ & $1(4.5)$ \\
\hline
\end{tabular}

COVID-19 - coronavirus disease 2019; $n$ - sample size; Cl - confidence interval; No. - number of patients; BMI - body mass index; CT - computed tomography

A
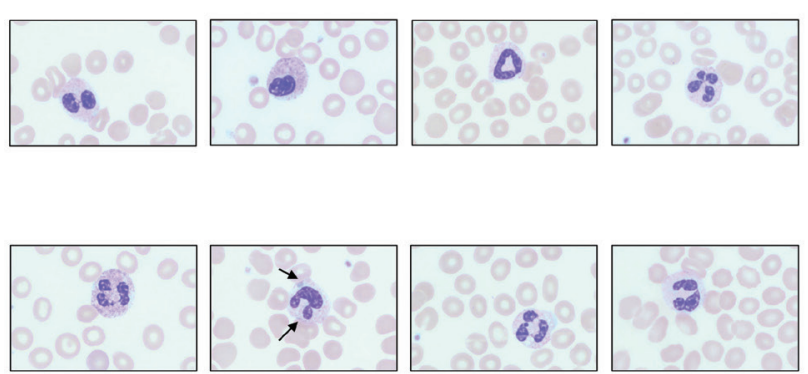

C

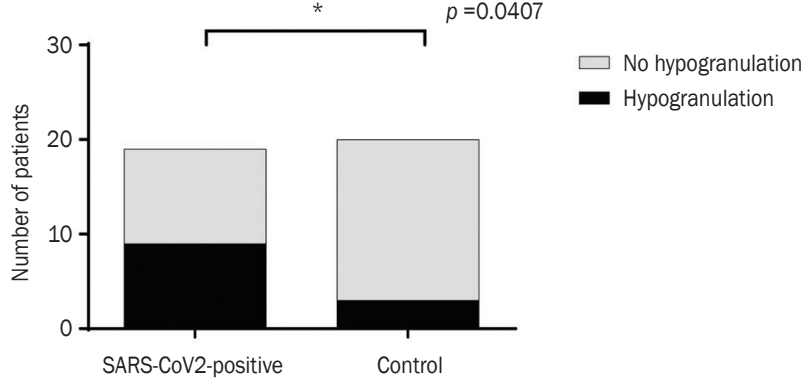

D
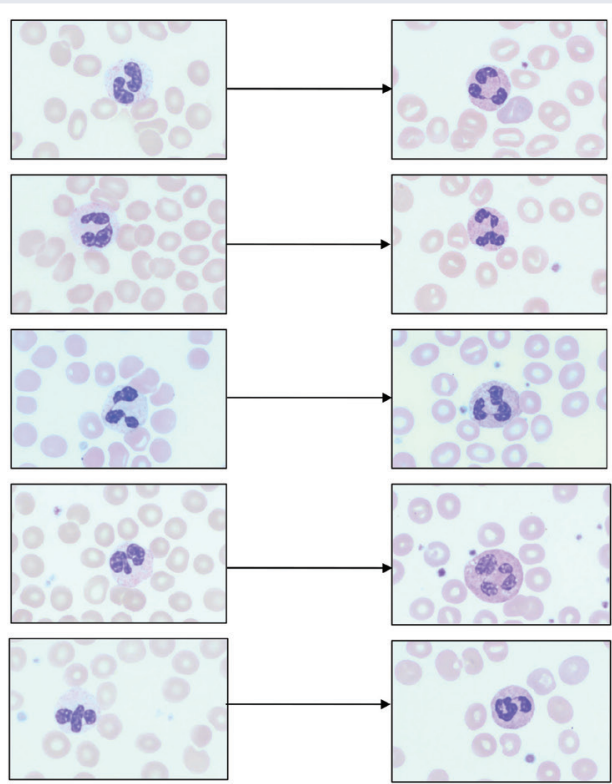

Figure 1A. Nuclear abnormalities in neutrophils with hyposegmented, ring-shaped and bizarre nuclei (from left to right); B. Cytoplasmic alterations including toxic granulations, Döhle bodies (arrows), cytoplasmic vacuoles and hypogranularity (from left to right); C. Comparison of hypogranularity in neutrophilic granulocytes in patients with severe acute respiratory syndrome coronavirus 2 (SARS-CoV-2) versus control patients at time of presentation to hospital; D. Five examples of reversible hypogranularity of neutrophilic granulocytes (patients P1-P5). Left: blood smears at diagnosis of coronavirus disease 2019. Right: matching blood smears during recovery time in hospital 


\section{Acknowledgements}

The authors wish to thank all patients and referring physicians for their support.

\section{Authors' contributions}

CR, CM, MG, SG, RE, TT and IK acquired data. CR and CM analyzed data. $C R, E M, A N$ and $C M$ wrote draft manuscript. $\mathrm{AB}, \mathrm{CK}, \mathrm{HR}$ and $\mathrm{CS}$ worked on manuscript. All authors agreed to content of manuscript.

\section{Conflict of interest}

For CS: Consultancy and research funding: Hycor Biomedical, Bencard Allergie and Thermo Fisher Scientific; Research funding: Mead Johnson Nutrition (MJN).

\section{Financial support}

This study was supported by the German José Carreras Leukemia Foundation (AH 06-01:AN) and the „EFRE-Förderung für Pandemie- und Pandemiefolgenbekämpfung im Rahmen der Investitionsinitiative Plus zur Bewältigung der Coronakrise" (project no. 62033243)... CS is supported by the Universities of Giessen and Marburg Lung Center (UGMLC), the German Center for Lung Research (DZL), University Hospital Giessen and Marburg (UKGM) research funding according to article 2, section 3 cooperation agreement, and the Deutsche Forschungsgemeinschaft (DFG)-funded SFB 1021 (C04), KF0 309 (P10), and SK 317/1-1 (Project number 428518790) as well as by the Foundation for Pathobiochemistry and Molecular Diagnostics.

\section{Ethics}

The work described in this article has been carried out in accordance with The Code of Ethics of the World Medical Association (Declaration of Helsinki) for experiments involving humans; EU Directive 2010/63/EU for animal experiments; Uniform requirements for manuscripts submitted to biomedical journals.

\section{References}

1. Chen N, Zhou M, Dong X, et al. Epidemiological and clinical characteristics of 99 cases of 2019 novel coronavirus pneumonia in Wuhan, China: a descriptive study. Lancet. 2020; 395(10223): 507-513, doi: 10.1016/S0140-6736(20)30211-7, indexed in Pubmed: 32007143.

2. Zhu Na, Zhang D, Wang W, et al. China Novel Coronavirus Investigating and Research Team. A novel coronavirus from patients with pneu- monia in China, 2019. N Engl J Med. 2020; 382(8): 727-733, doi: 10.1056/NEJMoa2001017, indexed in Pubmed: 31978945.

3. Terpos E, Ntanasis-Stathopoulos I, Elalamy I, et al. Hematological findings and complications of COVID-19. Am J Hematol. 2020; 95(7): 834-847, doi: 10.1002/ajh.25829, indexed in Pubmed: 32282949.

4. Jones JR, Ireland R. Morphological changes in a case of SARS-CoV-2 infection. Blood. 2020; 135(25): 2324, doi: 10.1182/ blood.2020006665, indexed in Pubmed: 32556139.

5. Lüke F, Orsó E, Kirsten J, et al. Coronavirus disease 2019 induces multi-lineage, morphologic changes in peripheral blood cells. EJHaem. 2020 [Epub ahead of print], doi: 10.1002/jha2.44, indexed in Pubmed: 32838398.

6. Zini G, Bellesi S, Ramundo F, et al. Morphological anomalies of circulating blood cells in COVID-19. Am J Hematol. 2020; 95(7): 870-872, doi: 10.1002/ajh.25824, indexed in Pubmed: 32279346.

7. Lee CT, Teo WZY. Peripheral blood smear demonstration of lymphocyte changes in severe COVID-19. Am J Trop Med Hyg. 2020; 103(4): 1350-1351, doi: 10.4269/ajtmh.20-0721, indexed in Pubmed: 32783797.

8. Nazarullah A, Liang C, Villarreal A, et al. Peripheral blood examination findings in SARS-CoV-2 infection. Am J Clin Pathol. 2020; 154(3): 319-329, doi: 10.1093/ajcp/aqaa108, indexed in Pubmed: 32756872.

9. Huang $C$, Wang $Y$, Li X e et al. Clinical features of patients infected with 2019 novel coronavirus in Wuhan, China. Lancet. 2020; 395(10223): 497-506, doi: 10.1016/S0140-6736(20)30183-5, indexed in Pubmed: 31986264.

10. Wang D, Hu Bo, Hu C, et al. Clinical characteristics of 138 hospitalized patients with 2019 novel coronavirus-infected pneumonia in Wuhan, China. JAMA. 2020; 323(11): 1061-1069, doi: 10.1001/ jama.2020.1585, indexed in Pubmed: 32031570.

11. Wu C, Chen X, Cai Y, et al. Risk factors associated with acute respiratory distress syndrome and death in patients with coronavirus disease 2019 pneumonia in Wuhan, China. JAMA Intern Med. 2020; 180(7): 934-943, doi: 10.1001/jamainternmed.2020.0994, indexed in Pubmed: 32167524.

12. Veras FP, Pontelli MC, Silva CM, et al. SARS-CoV-2-triggered neutrophil extracellular traps mediate CoVID-19 pathology. J Exp Med. 2020; 217(12), doi: 10.1084/jem.20201129, indexed in Pubmed: 32926098.

13. Gustine JN, Jones D. Immunopathology of hyperinflammation in COVID-19. Am J Pathol. 2021; 191(1): 4-17, doi: 10.1016/j.ajpath.2020.08.009, indexed in Pubmed: 32919977.

14. Berber I, Cagasar O, Sarici A, et al. Peripheral blood smear findings of COVID-19 patients provide information about the severity of the disease and the duration of hospital stay. Mediterr J Hematol Infect Dis. 2021; 13(1): e2021009, doi: 10.4084/MJHID.2021.009, indexed in Pubmed: 33489048.

15. Lacy P. Mechanisms of degranulation in neutrophils. Allergy Asthma Clin Immunol. 2006; 2(3): 98-108, doi: 10.1186/1710-1492-2-3-98, indexed in Pubmed: 20525154. 